investigators used a tactile PPI paradigm that involved administration of puffs of air to the participants. During the behavioral testing, the eye-blink component of the startle response was measured using electromyography, and participants underwent functional MRI to assess which areas of the brain were activated during PPI.

The PPI of the eye-blink startle response was reduced in patients compared with controls, although the reduction was milder for those patients receiving atypical antipsychotics. With PPI, control subjects showed increased activity in the striatum, thalamus, insula and hippocampus, and in the temporal, inferior frontal and inferior parietal regions of the brain. Overall, patients with schizophrenia showed reduced levels of activation in the thalamus, insula and inferior frontal regions compared with controls, but this was most pronounced for patients receiving typical antipsychotics.

The authors suggest that their results provide preliminary evidence that atypical antipsychotics can reduce both the PPI deficit and the associated brain abnormalities in patients with schizophrenia.

Original article Kumari V et al. (2006) A fMRI investigation of startle gating deficits in schizophrenia patients treated with typical or atypical antipsychotics. Int J Neuropsychopharmacol [doi: 10.1017/S1461145706007139]

\section{Large study finds association between SNCA promoter variability and PD}

Several small studies have suggested that certain alleles of a dinucleotide repeat sequence (REP1) of the $\alpha$-synuclein (SNCA) promoter might be associated with the risk of developing Parkinson's disease (PD). A large-scale collaborative study, involving 18 genetic consortium sites around the world, was set up to test this hypothesis.

Data on the three most common REP1 alleles (259 bp, 261 bp and 263 bp) were collected. Frequency data for the haplotypes defined by the REP1 loci, and for two single-nucleotide polymorphisms flanking the SNCA promoter at the -770 and -116 loci, were also gathered.

Complete data sets for 2,692 cases of PD and 2,652 unrelated controls were obtained from the 11 sites that met data bias and sensitivity standards. Genotypes defined by the 263 bp
REP1 allele had a significantly increased risk of developing PD compared with genotypes lacking this allele $(P<0.001)$. By contrast, genotypes including the $259 \mathrm{bp}$ allele had a significantly reduced risk of developing PD $(P=0.002$ for trend). Surprisingly, no association was found between any of the REP1 alleles and age at onset of PD. The frequencies of the REP1, -770 and -116 loci in individuals with PD were significantly different from those in individuals without PD $(P<0.001)$; further analysis revealed that the REP1 locus was responsible for the association between haplotype and risk of PD. The authors conclude that in the general population, variability at the SNCA REP1 locus might account for approximately $3 \%$ of the risk of developing PD.

Original article Maraganore DM et al. (2006) Collaborative analysis of $\alpha$-synuclein gene promoter variability and Parkinson disease. JAMA 296: 661-670

\section{Early treatment of schizophrenia normalizes metabolic profile}

No empirical disease markers of schizophrenia currently exist, rendering the diagnosis of this condition a subjective process and the development of presymptomatic treatments difficult. Results of a study investigating the metabolic profiles of cerebrospinal fluid (CSF) from patients with schizophrenia now indicate that drug-naive patients have altered levels of CSF glucose that can be normalized if treatment commences early.

Holmes et al. collected 152 CSF samples from drug-naive patients with first-onset paranoid schizophrenia, and carried out metabolic profiling of the samples using ${ }^{1} \mathrm{H}$ nuclear magnetic resonance spectroscopy. Analysis was also undertaken on CSF samples from healthy volunteers and from patients with schizophrenia who had experienced several psychotic episodes. Patients were given shortterm treatment with either a typical or an atypical antipsychotic drug, after which their CSF metabolic profiles were re-examined.

Drug-naive patients with first-onset schizophrenia had significantly elevated CSF glucose concentrations in comparison with healthy controls $(P=0.005)$. After short-term treatment with an atypical antipsychotic, however, the metabolic profile of approximately $50 \%$ of 